\title{
Pre-Test Probability of Coronary Artery Disease
}

National Cancer Institute

\section{Source}

National Cancer Institute. Pre-Test Probability of Coronary Artery Disease. NCI

Thesaurus. Code C80416.

An estimate of the likelihood that a patient has coronary artery disease prior to any testing. 